\title{
Pseudodifferential Operators on Supermanifolds and the Atiyah-Singer Index Theorem ${ }^{\star}$
}

\author{
Ezra Getzler
}

Department of Mathematics, Harvard University, Cambridge, MA 02138, USA

\begin{abstract}
Fermionic quantization, or Clifford algebra, is combined with pseudodifferential operators to simplify the proof of the Atiyah-Singer index theorem for the Dirac operator on a spin manifold.
\end{abstract}

\section{Introduction}

Recently, an outline of a new proof of the Atiyah-Singer index theorem has been proposed by Alvarez-Gaumé [1], extending unpublished work of Witten. He makes use of a path integral representation for the heat kernel of a Hamiltonian that involves both bosonic and fermionic degrees of freedom. In effect, a certain democracy is created between the manifold and the fermionic variables corresponding to the exterior algebra $\Lambda^{*} T_{x}^{*} M$ at each point $x \in M$. In this paper, this idea is pursued within the context of Hamiltonian quantum mechanics, for which the powerful calculus of pseudodifferential operators exists, permitting a rigorous treatment of Alvarez-Gaumé's ideas.

Most of the work will be to unify pseudodifferential operators with their fermionic equivalent, the sections of a Clifford algebra bundle over the manifold. Using a pseudodifferential calculus based on the papers of Bokobza-Haggiag [7] and Widom $[17,18]$, but incorporating a symbol calculus for the Clifford algebra as well, an explicit formula for composition of principal symbols is derived (0.7). This permits a complete calculation of the index of the Dirac operator to be performed, modeled on a proof of Weyl's theorem on a compact Riemannian manifold $M$.

Recall that this theorem states that

$$
\operatorname{Tr} e^{-t \Delta}=\left(\frac{\pi}{t}\right)^{n / 2} \operatorname{vol}(M)+O\left(t^{-n / 2+1}\right) .
$$

Using the calculus of pseudodifferential operators, one shows that the symbol $\sigma$ of the heat kernel satisfies.

$$
\sigma\left(e^{-t \Delta}\right)=e^{-t|\xi|^{2}}+\text { small error, }
$$

\footnotetext{
* Supported in part by the National Science Foundation under Grant No. PHY82-03669
} 
where $|\xi|^{2}$ is in fact the symbol of $\Delta$.

Combining (0.2) with a formula for the trace of a pseudodifferential operator

$$
\operatorname{Tr} P=\int_{T^{*} M} \sigma(P) d x d \xi
$$

the theorem follows, by evaluating the Gaussian integrals along each fibre.

Of course, we have not specified what we mean by the full symbol of a pseudodifferential operator for a Riemannian manifold. We use the exponential map of $M$ to pull back the pseudodifferential operator near $x \in M$ to $T_{x} M$, and calculate its symbol on $T_{x}^{*} M$ using the Euclidean structure. For details of the above proof, refer to the paper of Widom [17].

We prove the Atiyah-Singer theorem for the Dirac operators on a spin manifold. The proof extends in an obvious fashion to $\operatorname{spin}^{c}$ manifolds, so also provides a proof of the Riemann-Roch-Hirzebruch theorem. Moreover, the $\operatorname{spin}^{c}$ index theorem, combined with Bott periodicity, suffices to prove the full Atiyah-Singer index theorem.

The formalism of this paper may be cast in the language of supermanifolds, which is of increasing interest to mathematicians (Leites [15] and Witten [19]). From that point of view, this paper investigates an algebra of pseudodifferential operators with symbols that are $C^{\infty}$ functions on a symplectic supermanifold, rather than on $T^{*} M$. The most notable result here is the formula for the trace of a pseudodifferential operator (Theorem 3.7), which is formally similar to the formula (0.3). The remainder of the introduction interprets these analogies. However, the proof itself has been written in the standard languages of differential geometry and pseudodifferential operators, and is independent of any theories of supermanifolds.

The major ingredient of the above proof of Weyl's theorem was the symplectic manifold $T^{*} M$ and quantization on it, which relates suitable classical symbols in $C^{\infty}\left(T^{*} M\right)$ with operators on $C^{\infty}(M)$. We replace $T^{*} M$ by a symplectic supermanifold $\mathscr{N}$ that is associated with any Riemannian manifold $M$, has underlying manifold $T^{*} M$, and whose $C^{\infty}$ functions are sections of $\pi^{*}\left(\Lambda^{*} M\right)$, the pullback of $\Lambda^{*} M=\Lambda^{*} T^{*} M$ to $T^{*} M$. In a sense, $\mathcal{N}$ is the cotangent superbundle of the "supermanifold" $\mathscr{M}$ with $C^{\infty}(\mathscr{M})=\Gamma(\Delta)$, where $\Delta$ is a spinor bundle on $M$-except that $\mathscr{M}$ is not a supermanifold (except if, say, $M$ is a complex manifold, for which the spinor bundle is $\Lambda^{0, *} M$, which has a canonical graded algebra structure). Nevertheless, it is useful to think of $\mathscr{N}$ as some sort of cotangent bundle.

To prove that $\mathscr{N}$ is a symplectic supermanifold, recall that a symplectic structure on the superspace $V=V_{0} \oplus V_{1}$ is given by a symplectic structure on $V_{0}$ and a nondegenerate inner product on $V_{1}$. In our case, $V_{0}=W \oplus W^{*}$ and $V_{1}=W^{*}$; we impose the canonical symplectic structure on $V_{0}$ and use the Riemannian structure of $M$ to give an inner product to $V_{1}$.

The supermanifold $\mathscr{N}$ is a fibre bundle over $T^{*} M$, with structure group $O(n)$, where $\operatorname{dim} M=n$, and principal bundle $\pi^{*} O(M)$. In view of the diagram

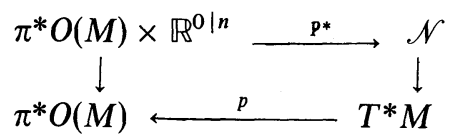


it is sufficient to construct a closed 2-form on $\pi^{*} O(M) \times \mathbb{R}^{0 \mid n}$ which is equivariant under the action of $O(n)$, on the right for $\pi^{*} O(M)$ and on the left for $\mathbb{R}^{0 \mid n}$, and is degenerate only along this action. ( $\mathbb{R}^{0 / n}$ is the superspace with $n$ odd coordinates.) If $\omega_{0}$ is the symplectic form on $T^{*} M$, and $g$ is the usual inner product on $\mathbb{R}^{0 \mid n}$, which is a closed 2-form on $\mathbb{R}^{0 \mid n}$, then we define our closed 2-form to be

$$
\omega=p^{*} \omega_{0}+g .
$$

Hence this descends to a symplectic form on $\mathscr{N}$. This supermanifold has been used, with $M=\mathbb{R}^{n}$, by Berezin and Marinov [6] to describe a classical spinning particle.

Just as for an ungraded symplectic manifold, one may define a Poisson bracket on $C^{\infty}(\mathcal{N})$ that gives it the structure of a super Lie algebra. We would like to associate functions in $C^{\infty}(\mathcal{N})$ with pseudodifferential operators on $\mathscr{M}$, the "base space" of $\mathscr{N}$. Returning once more to the linear setting, we recall that linear quantization leads to the following correspondences.

Table 1

Even Odd

\begin{tabular}{llll}
\hline \multirow{2}{*}{ Classical } & Phase space & Symplectic vector space $\mathbb{R}^{2 n}$ & $\begin{array}{l}\text { Non-degenerate inner product } \\
\text { space } \mathbb{R}^{2 n} \\
p: \operatorname{SOO}(2 n) \rightarrow \operatorname{End}\left(\mathbb{R}^{2 n}\right) \\
\text { Exterior polynomials } \Lambda^{*} \mathbb{R}^{2 n}\end{array}$ \\
\hline \multirow{3}{*}{ Quantum } & Observables & $\begin{array}{l}p: \operatorname{Sp}(n) \rightarrow \operatorname{End}\left(\mathbb{R}^{2 n}\right) \\
\text { Polynomials } V^{*} \mathbb{R}^{2 n}\end{array}$ & $\begin{array}{l}\text { Spinors } \Delta=\Lambda^{*} \mathbb{R}^{n} \\
p: \text { Spin }(2 n) \rightarrow U\left(\Lambda^{*} \mathbb{R}^{n}\right) \\
\text { Clifford algebra } C\left(\mathbb{R}^{2 n}\right)\end{array}$ \\
& Hilbert space & Symplectic spinors $L^{2}\left(\mathbb{R}^{n}\right)$ & $\begin{array}{l}p: M p(n) \rightarrow U\left(L^{2}\left(\mathbb{R}^{n}\right)\right) \\
\text { Polynomial coefficient } \\
\text { differential operators }\end{array}$ \\
& Observables & $\mathbb{C}\left[x_{1}, \ldots, x_{n}, \frac{\partial}{\partial x_{1}}, \ldots, \frac{\partial}{\partial x_{n}}\right]$ &
\end{tabular}

The right column, describing the theory of spinors and Clifford algebras, is due to Brauer and Weyl [10], and will be reviewed in the next section. The left column achieved its final form in Kostant's theory of symplectic spinors (Kostant [14]). The point is that in a super context, the two columns are merged into one, and it is seen that the Clifford algebra of an inner product space plays the role of the pseudodifferential operator algebra, while the exterior algebra plays the role of symbol space.

This example makes it natural to define the algebra of pseudodifferential operators on a supermanifold by combining the pseudodifferential operators on the base manifolds with sections of a Clifford algebra bundle. In the case of $\mathscr{N}$, for which $C^{\infty}(\mathcal{N})=C^{\infty}\left(T^{*} M\right) \otimes \Gamma\left(\Lambda^{*} M\right)$, we choose as our algebra of symbols $\mathscr{S}^{*}=S^{*}(M) \otimes \Gamma\left(\Lambda^{*} M\right)$; for the definition of $S^{*}(M) \subset C^{\infty}\left(T^{*} M\right)$, which is the class of classical symbols, see Sect. 2. Similarly, the algebra of pseudodifferential operators is $\mathrm{Op} \mathscr{S}^{*}=\mathrm{Op} S^{*}(M) \otimes \Gamma(C(M))$, and we will define a symbol map $\sigma$ and a quantization map $\theta$, which are almost inverses: 


$$
\mathscr{S}^{*} \underset{\sigma}{\stackrel{\theta}{\rightleftarrows}} \mathrm{Op} \mathscr{S}^{*}
$$

The operators in Op $\mathscr{S}^{*}$ act on the Hilbert space of $L^{2}$ sections of the spinor bundle $\Delta$ of $M$, with common dense domain $\Gamma(\Delta)$, the $C^{\infty}$ sections.

For the application to the index theorem, it turns out to be suitable to give $\mathscr{S}^{*}$ the filtration such that

$$
\mathscr{S}^{m}=\sum_{j+k=m} S^{j}(M) \otimes \Gamma\left(\Lambda^{k} M\right)
$$

This induces a corresponding filtration on $\mathrm{Op} \mathscr{S}^{m}$, and we will show that

$$
\text { Op } \mathscr{S}^{m}{ }_{\circ} \mathrm{Op} \mathscr{S}^{n} \subset \mathrm{Op} \cdot \mathscr{S}^{m+n},
$$

and also that the following remarkable formula for the top order symbol is true with the above filtration of $\mathscr{S}^{*}$ : if $p \circ q=\sigma(\theta p \circ \theta q)$, for $p, q \in \mathscr{S}^{*}$, then (Theorem 3.5)

$$
p \circ q(x, \xi)=\left.e^{-\frac{1}{4} R(\partial / \xi \xi, \partial / \partial)} p(x, \xi) \wedge q(x, \eta)\right|_{\xi=\eta}+\text { lower order terms. }
$$

Notice that the curvature operator $R(\partial / \partial \xi, \partial / \partial \eta)$ does not change the degree when applied to $p \wedge q$ : the two derivatives lower the degree by two, but this is cancelled by the curvature $R$ having degree two. This formula may be compared to the formulas for the top order of the composition of symbols in other classes of pseudodifferential operators, for instance, Boutet de Monvel's classes $S^{p, k}$ which degenerate along a submanifold of $T^{*} M$ (Boutet de Monvel [8]). Like these cases, one obtains here differential equations on a set of linear spaces (the fibres of $T^{*} M$ ), which are of course much easier to handle than the full equation.

We will apply this formula to the square of the Dirac operator, $\mathscr{D}^{2}$, which has top order symbol $-|\xi|^{2}$. If $\operatorname{Tr}_{s}$, the supertrace, is defined as $\operatorname{Tr}\left|V_{0}-\operatorname{Tr}\right| V_{1}$ for a superspace $V=V_{0} \oplus V_{1}$, then the index of $\mathscr{D} \mid \Gamma\left(\Delta^{+}\right)$is equal to $\operatorname{Tr}_{s} e^{t \mathscr{D}^{2}}$ for any $t>0$. This is reminiscent of $\operatorname{Tr} e^{-t \Delta}$, which is calculated by Weyl's theorem. Our proof of the index theorem may be thought of as a superspace version of Weyl's theorem.

By $(0.7),|\xi|^{2} \circ p=\left(|\xi|^{2}-\frac{1}{2} R(\xi, \partial / \partial \xi)-\frac{1}{16} R \wedge R(\partial / \partial \xi, \partial / \partial \xi)\right) p$, up to lower order terms; we will show that as $t \rightarrow 0$, the symbol of $e^{t \mathscr{D}^{2}}$ is increasingly closely approached by $e^{-t\left(\mid \xi \xi^{2}-\frac{1}{2} R(\xi, \partial / \partial \xi)-\frac{1}{16} R \wedge R(\partial / \partial \xi, \partial / \partial \xi)\right)}$, analogously to formula $(0.2)$ for $\sigma\left(e^{-t \Delta}\right)$; this operator is understood to be applied to the constant symbol 1 on $T^{*} M$. This symbol may be calculated explicitly from Mehler's formula (2.10) for the heat kernel of the harmonic oscillator, since the exponent is essentially the harmonic oscillator with frequency ${ }^{-1}$ equal to the curvature $R($, ).

Finally, it is shown in Theorem 3.7 that the supertrace can be calculated by integrating the symbol over the supermanifold - explicitly, the part of the symbol in $S^{*}(M) \otimes \Gamma\left(\Lambda^{2 n} M\right)$ (where $M$ has dimension $2 n$ ) is projected out, and this is integrated over the cotangent space $M$. (A more invariant formulation may be derived from the fact that an oriented symplectic supermanifold has a canonical volume form, since $\operatorname{SOSp}(V) \hookrightarrow S L(V)$ for any superspace $V$.) This easily leads to the formula, for the index of $\mathscr{D}$ in terms of the $\hat{A}$-genus of $M$.

In fact, the theorem will be proved for the Dirac operator associated to a vector 
bundle $E$ on $M$. Nevertheless, the above calculation remains essentially unchanged, except for the appearance of the Chern character of $E$.

This proof may be considered to be an improved version of those of Patodi [16] and Atiyah, Bott and Patodi [3] in two senses: the use of Clifford algebras and their symbols eliminates the combinatorics of the earlier proofs, and shows that the cancellations which occur are not all that remarkable; also, this proof leads to the explicit formula for the Â-genus, while the earlier proofs had to appeal to various topological characterizations of the $\hat{A}$-genus.

In concluding this introduction, I would like to thank for many conversations and much stimulation, L. Alvarez-Gaumé, A. Jaffe, who suggested that I look at this problem, T. Parker, and R. Bott.

\section{Clifford Algebras and Spinors}

The facts about Clifford algebras and the spinor representation that we use are scattered widely through the literature (Atiyah and Bott [2], Bourbaki [9], Chevalley [11]). The following summary of the theory is fairly standard, and most of the proofs are omitted.

If $V$ is an inner product space, over $\mathbb{R}$ or $\mathbb{C}$, then the Clifford algebra of $V$, denoted $C(V)$, is the algebra generated by $V$ with the relations,

$$
v \cdot w+w \cdot v=2(v, w) .
$$

Thus $C(V)$ is the quotient of the tensor algebra of $V$ by the ideal $I$ generated by $\left\{v \otimes v-|v|^{2} \mid v \in V\right\}$. Since $I$ is invariant under the action of the orthogonal group $O(V)$, there is an action of $O(V)$ on $C(V)$ as an algebra extending its action on $V \subset C(V)$. Composing the injection of the exterior algebra $\Lambda^{*} V$ into the tensor algebra of $V$ with projection onto $C(V)$ gives an isomorphism of the $O(V)$ modules $\theta: \Lambda^{*} V \rightarrow C(V)$. This induces a filtration of $C(V)$, in which $C^{m}(V)=\sum_{j \leqq m} \theta\left[\Lambda^{j} V\right]$, which satisfies

$$
C^{j}(V) \cdot C^{k}(V) \subset C^{j+k}(V) .
$$

We think of $\theta$ as a quantization map that assigns to a symbol in $\Lambda^{*} V$ its operator in $C(V)$. If we define the symbol map to be $\sigma=\theta^{-1}$, then

$$
\sigma(a \cdot b)=\sigma(a) \wedge \sigma(b) \bmod \Lambda^{|a|+|b|-1} V .
$$

In fact, this follows from the full formula for $\sigma(a \cdot b)$,

$$
\sigma(a \cdot b)=\sigma(a) \exp (\overleftarrow{\partial}, \vec{\partial}) \sigma(b)
$$

In this formula, $\vec{\partial}: \Lambda^{*} V \rightarrow V \otimes \Lambda^{*} V$ is the adjoint of contraction on the left, $V^{*} \otimes \Lambda^{*} V \rightarrow \Lambda^{*} V ; \overleftarrow{\partial}: \Lambda^{*} V \rightarrow \Lambda^{*} V \otimes V$ is the adjoint of right contraction; $($, ) is the inner product on $V$; exterior multiplication is understood. This formula is easily checked in an orthonormal basis of $V$.

The Clifford algebra is a $\mathbb{Z}_{2}$-algebra; under the $\mathbb{Z}_{2}$-grading $C_{i}(V)=$ $\sum_{m=i \bmod 2} \theta\left[\Lambda^{m} V\right], i=0,1$, we have

$$
C_{i}(V) \cdot C_{j}(V) \subset C_{i+j}(V)
$$


The action of $O(V)$ on $C(V)$ leads to an injection of $o(V)$ into the Lie algebra $\operatorname{der}(C(V))$ of derivations of $C(V)$. Let $\left\{e_{i} \mid 1 \leqq i \leqq n\right\}$ be an orthonormal basis of $V$. If $\left(a_{i j}\right) \in O(V)$, so that $a_{i j}=-a_{j i}$, then it maps to $\operatorname{ad}\left(\frac{1}{2} \sum_{i<j} a_{i j} e_{i} e_{j}\right)$ in $\operatorname{der}(C(V))$ (this is easily proved by verifying it on $V \subset C(V)$ ). If this Lie algebra is exponentiated inside $C(V)$, a covering group $G \subset C(V)$ of $\mathrm{SO}(V)$ is obtained, with covering map given by Ad: $G \rightarrow \operatorname{Aut}(C(V))$. Since $e^{\pi e_{1} e_{2}}=-1 \in G$, it follows if $\operatorname{dim} V \geqq 2$ that $G$ is a double covering of $\operatorname{SO}(V)$, so is simply connected and isomorphic with $\operatorname{Spin}(V)$.

We now give a brief discussion of the construction of the spinor representation of $C(V)$, assuming $V$ is an even dimensional complex vector space. (If $V$ is real, then the injection $V \rightarrow V \otimes \mathbb{C}$ induces an injection $C(V) \rightarrow$ $C(V \otimes \mathbb{C}) \cong C(V) \otimes \mathbb{C}$.

Let $P, Q$ be a maximal transverse pair of isotropic subspaces of $V$, that is, $P \cap Q=0,(P, P)=(Q, Q)=0$, and $P$ and $Q$ have dimension $n=\frac{1}{2} \operatorname{dim} V$. For instance, let $P$ (respectively $Q$ ) have the basis $e_{2 j-1}+i e_{2 j}$ (respectively $e_{2 j-1}-i e_{2 j}$ ). The inner product on $V$ places $P$ and $Q$ in duality.

Let $\Delta=A^{*} P \subset C(V)$ (by the inclusion $P \subset V$ ). We let $V$ act on $\Delta$ by

$$
\begin{cases}p \cdot a=\sqrt{2} p \wedge a, & p \in P, a \in \Delta, \\ q \cdot a=\sqrt{2} q\lrcorner a, & q \in Q .\end{cases}
$$

This action extends to an isomorphism of $C(V)$ with End $(\Delta)$. The space $\Delta$ is called the space of spinors, and if $\Delta$ is given the $\mathbb{Z}_{2}$-grading $\Delta^{+}=\Lambda^{\mathrm{ev}} p, \Delta^{-}=\Lambda^{\text {od }} p$, then it is a graded module for $C(V)$ :

$$
C_{0}(V) \cdot \Delta^{ \pm} \subset \Delta^{ \pm}, C_{1}(V) \cdot \Delta^{ \pm} \subset \Delta^{ \pm} .
$$

Let $\omega=i^{n} e_{1} \ldots e_{2 n} \in C_{0}(V)$, so that $\omega^{2}=1$ and $\sigma(\omega)$ is (up to a factor of $i^{n}$ ) the volume form of $V$. The operator $\omega$, acting on $\Delta$, equals $\operatorname{Id}\left|\Delta^{+}-\operatorname{Id}\right| \Delta^{-}$; it is better known as $\gamma_{5}$ to physicists. Let $\operatorname{Tr}_{s} a$ be the trace of $a \in C(V)$ on $\Delta^{ \pm}$, and let $\operatorname{Tr}_{s} a=\operatorname{Tr}_{+} a-\operatorname{Tr}_{-} a$ (the $s$ stands for super).

\section{Theorem 1.8 .}

i) $2^{n}(\operatorname{Tr} a)$ equals the projection of $\sigma(a) \in \Lambda^{*} V$ onto $\Lambda^{0} V \cong \mathbb{C}$.

ii) $2^{n}\left(\operatorname{Tr}_{s} a\right)$ equals the projection of $\sigma(a) \in \Lambda^{*} V$ onto $\Lambda^{2 n} V$, identified with $\mathbb{C}$ by the map $\omega \mapsto 1$.

Proof. a) If $I=\left\{I_{1}<\ldots<I_{m}\right\} \subset\{1, \ldots, 2 n\}$, then let $e_{I}=e_{I_{1} \ldots I_{m}}$. As $I$ varies over all subsets of $\{1, \ldots, 2 n\}$, the vectors $e_{I} \in C(V)$ form a basis of $C(V)$. If $|I|$ is odd, then $e_{I} \cdot \Delta^{ \pm} \subset \Delta^{\mp}$ so $\operatorname{Tr} e_{I}=0$. If $|I|$ is even and $I \neq \emptyset$, then for some $i \in I$,

$$
\operatorname{Tr} e_{I}= \pm \operatorname{Tr}\left(\left[e_{i}, e_{I \backslash\{i\}}\right]\right)=0 .
$$

Thus only multiples of $e_{\emptyset}=1$ contribute to $\mathrm{Tr}$.

b) This follows from $a$ ), since $\operatorname{Tr}_{s} a=\operatorname{Tr} a \omega$. 


\section{A Symbol Calculus on Spin Manifolds}

In order to calculate the index of the Dirac operator, we will develop a calculus for pseudodifferential operators on the spin bundle which is intimately related to the geometry of the manifold. This calculus is modeled on those of Bokobza-Haggiag [7] and Widom [17,18]; the form in which it is presented here is a synthesis of these two accounts, modified by the presence of the Clifford algebra.

The idea is as follows: zeroth order differential operators on the spin bundle $\Delta$ are given by sections of $\operatorname{End}(\Delta)$. By the results of the last section, this bundle is canonically isomorphic with $\Lambda^{*} M$, so we can filter these operators by their degree as a section of $\Lambda^{*} M$, that is, the degree of their symbols. We would then like to extend this filtration to all of the pseudodifferential operators on $\Delta$ assigning an operator the sum of its degree as a spinor endomorphism and its degree as a pseudodifferential operator in the usual sense.

To do this in some canonical way (since as stated, the assignment of degree is coordinate dependent), we recall the definition of the full symbol on a Riemannian manifold. For simplicity, we assume that $M$ is compact, although the calculus may be extended easily to arbitrary manifolds. The exponential map is written exp: $T M \rightarrow M \times M$, so that $\left(x \in M, v \in T_{x} M\right) \mapsto\left(x, \exp _{x} v\right)$.

Let $\alpha \in C^{\infty}(M \times M)$ satisfy the assumptions:

i) $\exp ^{-1}$ is a diffeomorphism in a neighbourhood of supp $\alpha$;

ii) $\alpha=1$ in a neighbourhood of the diagonal in $M \times M$.

The function $\alpha(x, \cdot)$ will be used to localize function on $M$ to a neighborhood of $x$ for which normal coordinates exist.

For any complex vector bundle $E$ with connection, over $M$, parallel translation from $x$ to $y$ along the geodesic between $x$ and $y$ is written as $\tau_{E}(x, y)$, at least for $(x, y) \in \operatorname{supp} \alpha$. We let $\alpha_{E}(x, y)=\alpha(x, y) \tau_{E}(x, y)$.

We will use the standard symbol classes: If $E$ and $F$ are two complex vector bundles over $M$, and $\pi$ is the projection $T^{*} M \rightarrow M$, then

$$
\begin{aligned}
S^{m}(E, F)= & \left\{p \in \Gamma\left(\pi^{*} \operatorname{Hom}(E, F)\right) \mid\right. \\
& \left|\partial_{x}^{\alpha} \partial_{\xi}^{\beta} p(x, \xi)\right| \leqq C_{\alpha \beta}(1+|\xi|)^{m-|\beta|} \quad \text { for all } \alpha, \beta \geqq 0, \\
& \text { where } \left.C_{\alpha \beta}>0\right\} .
\end{aligned}
$$

Here, $(x, \xi)$ are the usual coordinates on $T^{*} M$. In the applications, we will have $E=F$, although this restriction may be dispensed with.

To incorporate the spin bundle, we notice that

$$
S^{*}(\Delta \otimes E) \cong S^{*}(E) \underset{C^{\infty}(M)}{\otimes} \Gamma(\operatorname{End}(\Delta)) \cong S^{*}(E) \underset{C^{\infty}(M)}{\otimes} \Gamma\left(\Lambda^{*} M\right) .
$$

Using this realization of the symbols of pseudodifferential operators on $\Delta \otimes E$, we make the fundamental choice of filtration:

$$
\mathscr{S}^{m}(E)=\sum_{j+k=m} S^{j}(E) \underset{C^{\infty}(M)}{\otimes} \Gamma\left(\Lambda^{k} M\right) .
$$

The justification for this choice will become clearer later. 
It is now possible to define a quantization, which associates a pseudodifferential operator to each symbol, in an almost canonical way (that is, up to the choice of $\alpha$ ). In fact, if $u \in \Gamma(\Delta \otimes E)$, then $u_{x}=\exp _{x}^{*}\left(\alpha_{\Delta \otimes E}(x, y) u(y)\right)$ is in $C_{0}^{\infty}\left(T_{x} M\right) \otimes \Delta_{x} \otimes E_{x}$ for each $x \in M$, while if $p \in \mathscr{S}^{m}(E)$, then $\left.p\right|_{T_{M}^{*}} \in \mathscr{S}^{\prime}\left(T_{x}^{*} M\right) \otimes$ $\Lambda^{*} T_{x}^{*} M \otimes \operatorname{End}\left(E_{x}\right)$, and we can use the Fourier transform to produce a pairing between the two objects:

$$
(\theta p) u(x)=(2 \pi)^{-n} \int_{T_{x} M \times T_{x}^{*} M} e^{-i\langle v, \xi\rangle} p(x, \xi) u_{x}(v) d v d \xi .
$$

Clearly, the quantized operator $\theta p$ is bounded on $\Gamma(\Delta \otimes E)$; the set of such operators, supplemented by the regularizing operators, is denoted by Op $\mathscr{S}^{m}(E)$. As usual, we define $\mathscr{S}^{-\infty}=\cap \mathscr{S}_{m \in \mathbb{Z}}$, so that $\operatorname{Op} \mathscr{S}^{-\infty}(E)$ is the set of all regularizing operators. These are included so that $\operatorname{Op} \mathscr{S}^{*}(E)$ will be closed under composition.

The symbol map defines a symbol $\sigma P \in \mathscr{S}^{m}$ for each $P \in \mathrm{Op} \mathscr{S}^{m}$ (this will be proved later), and is defined as follows: $(\sigma P)(x, \xi)$ is the endomorphism of $\Delta_{x} \otimes E_{x}$, or member of $\Lambda^{*} T_{x}^{*} M \otimes \operatorname{End}\left(E_{x}\right)$, such that if $u \in \Delta_{x} \otimes E_{x}$, then

$$
(\sigma P)(x, \xi) u=\left.P_{y}\left(e^{i\left\langle\exp _{x}^{-1} y, \xi\right\rangle} \alpha_{\Delta \otimes E}(y, x) u\right)\right|_{x} .
$$

To provide some justification for these definitions, and before dealing with the technical difficulties that they give rise to, it is of interest to investigate their behavior on differential operators, which are of course better behaved. One of our goals is a formula for the top order symbol of the composition of two operators, and solving this problem for differential operators is already non-trivial. In fact, the formula will be relatively easy to extend to all pseudodifferential operators once it is known in this special case.

Let $\mathscr{P}^{m}(E)$ be the subset of $\mathscr{S}^{m}(E)$ consisting of all symbols that are polynomials in $\xi$. It is a straightforward consequence of Fourier theory that $\mathrm{Op} \mathscr{P} *(E)$, the image under $\theta$ of $\mathscr{P}^{*}(E)$, is exactly the set of all differential operators of the bundle $\Delta \otimes E$, and that $\sigma$ is a left and right inverse of $\theta$. Since this is independent of $\alpha$, the calculus is canonical for differential operators.

\section{Example 2.3}

a) If $X \in \Gamma(T M)$ is a vector field on $M$, then $\nabla_{X}$, covariant differentiation on $\Delta \otimes E$, is a differential operator. In a synchronous frame around $x$, that is, parallel along the geodesic rays outwards from $x$ in normal coordinates, the connection of $\Delta \otimes E$ vanishes at the point $x$. This shows that

$$
\left.\sigma\left(\nabla_{X}\right)=i X\right\lrcorner \xi \text {. }
$$

b) If $M$ has curvature $R \in \Gamma\left(\Lambda^{2} M \otimes \Lambda^{2} M\right) \subset S^{2}(E) \otimes \Gamma\left(\Lambda^{2} M\right)$, and $E$ has curvature $F \in \Gamma\left(\operatorname{End}(E) \otimes \Lambda^{2} M\right) \subset S^{0}(E) \otimes \Gamma\left(\Lambda^{2} M\right)$, then

$$
\left.\left.\left.\sigma\left(\nabla_{X} \circ \nabla_{Y}\right)=-(X\lrcorner \xi\right)(Y\lrcorner \xi\right)+\frac{1}{4} R(X, Y)+i \nabla_{X} Y\right\lrcorner \xi+\frac{1}{2} F(X, Y),
$$

where the first two terms are second order, the next is first order, and the last is zeroth order. Indeed, in normal coordinates at $x$, 


$$
\left.\nabla_{X} \circ \nabla_{Y}\right|_{2}=\left.X^{i}\left(\partial_{i}+\Gamma_{i}\right) Y^{j}\left(\partial_{j}+\Gamma_{j}\right)\right|_{x}=\left.X^{i} Y^{j}\left(\partial_{i} \partial_{j}+\Gamma_{j, i}\right)\right|_{x}+\left.X^{i}\left(\partial_{i} Y^{j}\right) \partial_{j}\right|_{x}
$$

In this formula, $\Gamma_{i}$ denotes the connection of $\Delta \otimes E$. We now make use of the formula, valid if $E$ is a bundle with connection $\Gamma$ and curvature $F$ and we are in a synchronous frame around $x: \Gamma_{j, i}(y)=F_{i j}(x)+O(|y-x|)$. The proof may be found in Atiyah, Bott and Patodi [3], Proposition 3.7. Furthermore, the curvature of $\Delta$ is half of the Riemannian curvature.

c) The Dirac operator $\mathscr{D}^{ \pm}$on $\Gamma\left(\Delta^{ \pm} \otimes E\right)$ is the composition of $\nabla: \Gamma\left(\Delta^{ \pm} \otimes E\right) \rightarrow$ $\Gamma\left(T^{*} M \otimes \Delta^{ \pm} \otimes E\right)$ with Clifford multiplication, which maps $T^{*} M \otimes \Delta^{ \pm}$to $\Delta^{\mp}$. With the spinor hermitian inner product on $\Delta^{ \pm}$, we have that the adjoint of $\mathscr{D}^{ \pm}$ is $-\mathscr{D}^{\mp}$, so that the index of $\mathscr{D}^{+}$is $\operatorname{dim} \operatorname{ker} \mathscr{D}^{+}-\operatorname{dim} \operatorname{ker} \mathscr{D}^{-}$. This is the integer that is to be computed by the Atiyah-Singer index theorem.

In Atiyah, Bott and Patodi [3], it is observed that

$$
\text { index } \mathscr{D}^{+}=\operatorname{Tr}_{s} e^{t \mathscr{D}^{2}} \text {, }
$$

where $t>0$ is arbitrary. Indeed, if $V_{\lambda}^{ \pm} \subset \Gamma\left(\Delta^{ \pm} \otimes E\right)$ is the eigenspace of $\mathscr{D}^{2}$ corresponding to the eigenvalue $\lambda \leqq 0$, then $\mathscr{D}^{+}: V_{\lambda}^{+} \rightarrow V_{\lambda}^{-}$is an isomorphism of vector spaces if $\lambda \neq 0$, so

$$
\operatorname{Tr}_{s} e^{a \mathscr{D}^{2}}=\operatorname{dim} V_{0}^{+}-\operatorname{dim} V_{0}^{-}=\operatorname{index} \mathscr{D}^{+} .
$$

In normal coordinates at $x \in M, \mathscr{D}=\sum e_{i} \nabla_{i}$, where $e_{i}$ is the action of $d x_{i} \in$ $\Gamma\left(T^{*} M\right)$ on $\Delta$. Thus,

$$
\begin{aligned}
\left.\mathscr{D}^{2}\right|_{x} & =\sum_{i j} e_{i} e_{j} \nabla_{i} \nabla_{j} \\
& =\sum_{i} \nabla_{i}^{2}+\frac{1}{4} \sum_{i j k l} R_{i j k l} e_{i} e_{j} e_{k} e_{l}+\frac{1}{2} \sum_{i j} F_{i j} e_{i} e_{j} .
\end{aligned}
$$

The second term is multiplication by $s / 4 \in \mathrm{Op} \mathscr{P}^{0}(E)$, by the Bianchi identity, where $s$ is the scalar curvature, so

$$
\sigma\left(\mathscr{D}^{2}\right)=-|\xi|^{2}+\frac{1}{2} F+\text { lower order. }
$$

We will now derive a formula for composing symbols in $\mathscr{P}^{*}(E)$. If $p \in \mathscr{P}^{k}(E)$ and $q \in \mathscr{P}^{l}(E)$, then define their composition to be

$$
p \circ q=\sigma(\theta p \circ \theta q) .
$$

It is not even obvious that $p \circ q \in \mathscr{P}^{k+l}(E)$; this follows from the following theorem.

Theorem 2.7. There exist differential operators $a_{n}, n \geqq 0$, on the bundle (over $\left.T^{*} M\right) \pi^{*}\left(\Lambda^{*} M \otimes \operatorname{End}(E)\right) \otimes \pi^{*}\left(\Lambda^{*} M \otimes \operatorname{End}(E)\right)$, such that if $a_{n}(p, q)$ denotes the exterior product on $\pi^{*}\left(\Lambda^{*} M \otimes \operatorname{End}(E)\right)$ applied to $a_{n}(p \otimes q)$, so that $a_{n}(p, q) \in \Gamma\left(\pi^{*}\left(\Lambda^{*} M \otimes \operatorname{End}(E)\right)\right)$, then

i) $a_{n}(p, q) \in \mathscr{P}^{k+l-n}(E)$;

ii) $p \circ q=\sum_{n=0}^{\infty} a_{n}(p, q)($ this is a finite sum);

iii) $a_{0}(p, q)(x, \xi)=\left.e^{-\frac{1}{4} R(\partial / \partial \xi, \partial / \partial \eta)} p(x, \xi) \wedge q(x, \eta)\right|_{\xi=\eta}$. 
Remark. If we had assigned to a $j^{\text {th }}$ order polynomial symbol in $\Lambda^{k} M$ the degree $j+c k$, with $c \geqq 0$, then i) and ii) remain true for $\frac{1}{2} \leqq c \leqq 1$. If $\frac{1}{2} \leqq c<1$, then $a_{0}(p, q)=p \wedge q$, just as in the usual calculus. However, we need the definition of degree given by $c=1$; the extra terms in $a_{0}(p, q)$ lead to the characteristic classes appearing in the formula for the index of $\mathscr{D}$.

Proof. Symbols of the type $h \in \Gamma(\operatorname{End}(E)), \omega \in \Gamma\left(\Lambda^{*} M\right)$ and pure polynomial in $\xi$, together generate $\mathscr{P}^{*}(E)$, so it suffices to prove the theorem when the two symbols are from one of these classes. If neither of the symbols depends on $\xi$, then the result follows from (1.4) which expresses Clifford multiplication in terms of exterior multiplication.

To handle polynomial symbols, we shall make use of the algebra of formal symbols $\left(\mathscr{P}^{*}(E)[[c]], \circ\right)$, where $c \in \mathbb{R}^{n}$. In normal coordinates around $x \in M$, let $c \cdot \xi=\sum c_{i} \xi_{i}$, and let $\exp (c \cdot \xi)$ denote the formal exponential of $c \cdot \xi$ in this algebra.

Lemma 2.8. There is an element $r \in \Gamma\left(\Lambda^{2} M \otimes \operatorname{End}(E)\right)[[c]]$ of order at least 3 in $c$, such that near $x$,

$$
\exp (c \cdot \xi)=(1+r) e^{c \cdot \xi}
$$

Proof. Let $X \in \Gamma(T M)$ be defined by $X=\sum c_{i} \partial_{i}$. In normal coordinates around $y$,

$$
\begin{aligned}
\left.\exp (c \cdot \xi)\right|_{y}= & \sigma(\exp (-i X\lrcorner(\partial+\Gamma)))\left.\right|_{y} \\
= & \sigma((1+\text { commutators of } X\lrcorner \partial \text { and } X\lrcorner \Gamma) \\
& \exp (-i X\lrcorner \partial))_{y}
\end{aligned}
$$

by the Campbell-Hausdorff formula, since $\left.\Gamma\right|_{y}=0$. An $m^{\text {th }}$ commutator of $\left.X\right\lrcorner \partial$ and $X\lrcorner \Gamma$ vanishes at $y$ for $m \leqq 2$, and has symbol in $\Gamma\left(\Lambda^{2} M \otimes \operatorname{End}(E)\right)$ for $m \geqq 3$; this uses the fact that $\Lambda^{2} M$ is a Lie algebra (isomorphic to $o(n)$ ) under the Clifford bracket.

To prove the theorem for $p \circ(\omega \otimes h)$, where $p$ is a polynomial in $\xi, \omega \in \Gamma\left(\Lambda^{k} M\right)$ and $h \in \Gamma(\operatorname{End}(E))$, it suffices (by expanding in $c$ ) to prove that $\sigma\left(e^{c \cdot \nabla} \circ(\theta \omega \otimes h)\right)$ $\omega \otimes h \otimes e^{\sigma(c \cdot \nabla)} \in \mathscr{P}^{*}(E)[[c]]$ is the sum of terms in $\mathscr{P}^{k+n}(E)[[c]]$ of order $\geqq n+1$ in $c$. Since covariant differentiation of a section of $C(M)$ gives the same answer as differentiating its symbol in $\Gamma\left(\Lambda^{*} M\right)$, and this preserves the degree of a differential form, the result follows.

In the following calculation, let $\cong$ denote equality modulo an element (or sum of such elements) in $\mathscr{P}^{n}(E)[[c]]$ of order $\geqq n+1$ in $C$. In this notation, Lemma 2.8 states that

$$
\exp (c \cdot \xi) \cong e^{c \cdot \xi}
$$

If $c, d \in \mathbb{R}^{u}$, then by the Campbell-Hausdorff formula,

$$
\begin{aligned}
e^{c \cdot \xi} \circ e^{d \cdot \xi} & \cong \sigma\left(e^{i c \cdot \nabla} \circ e^{i d \cdot \nabla}\right) \cong \sigma\left(e^{i(c+d) \cdot \nabla-\frac{1}{2}[c \cdot \nabla, d \cdot \nabla]}\right) \\
& \cong e^{-\frac{1}{4} R(c, d)} e^{(c+d) \cdot \xi}=\left.e^{-\frac{1}{4} R(\partial / \partial \xi, \partial / \partial \eta)} e^{c \cdot \xi} e^{d \cdot \eta}\right|_{\xi=\eta} .
\end{aligned}
$$

Expanding this formula in $c$ and $d$ proves the theorem if $p$ and $q$ are both pure polynomials in $\xi$, which is the last case which has to be considered. 
We will apply this formula in the case where $p=-|\xi|^{2}+\frac{1}{2} F$ is the symbol of $\mathscr{D}^{2}$ (neglecting lower terms). In this case, neglecting lower order terms,

$$
p \circ q(x, \xi)=\left(-|\xi|^{2}+\frac{1}{2} R\left(\xi, \frac{\partial}{\partial \xi}\right)+\frac{1}{16}(R \wedge R)\left(\frac{\partial}{\partial \xi}, \frac{\partial}{\partial \xi}\right)+\frac{1}{2} F\right) q(x, \xi) .
$$

If we ignore the second term for the moment, the remaining terms are related to the harmonic oscillator. This is where the formula for the index of $\mathscr{D}$ will come from: in the next section, we show how the symbol of the heat kernel for $\mathscr{D}^{2}$ is given by the heat kernel for the harmonic oscillator (with frequency ${ }^{-1}$ equal to the curvature). For now, we note the following formula, known as Mehler's formula (see Glimm, Jaffe [12]): if $k_{a}(x, y)$ is the kernel of $e^{-\frac{1}{2}\left(x^{2}-a^{2}\left(d^{2} / d x^{2}\right)\right)}$, the harmonic oscillator with frequency ${ }^{-1} a$ in one dimension, then

$$
k_{a}(x, y)=(2 \pi a \sinh a)^{-\frac{1}{2}} \exp \left(-\frac{1}{2 a \sinh a}\left(\cosh a\left(x^{2}+y^{2}\right)-2 x y\right)\right) .
$$

In particular, if $A$ is an antisymmetric matrix on $\mathbb{R}^{2 u}$, then the kernel of $e^{-H}$ satisfies

$$
\int k(x, y) d x d y=\prod_{j=1}^{n} \frac{2 \pi a_{j}}{\sinh a_{j}},
$$

where the eigenvalues of $A$ are $\left\{ \pm i a_{j} \mid 1 \leqq j \leqq n\right\}$. This is the Â-genus of the matrix $A$, which appears in the formula for the index of $\mathscr{D}$.

\section{Extension to Pseudodifferential Operators}

In this section, the formalism of the previous section is extended to pseudodifferential operators. Unlike for differential operators and polynomial symbols, the quantization map $\theta$ and the symbol map $\sigma$ are not inverses of each other in the spaces of pseudodifferential operators and their symbols. Widom [17] has developed a simple technique for avoiding this difficulty, which replaces $\theta$ and $\sigma$ by families of maps $\theta_{t}$ and $\sigma_{t}$, where $t>0$. As $t$ approaches zero, the symbol calculus becomes more accurate.

If $p \in \mathscr{S}^{m}(E)$ is a symbol, then for $t>0$, the dilated symbol $p_{t}$ is defined as follows: if $p=\sum_{j=0}^{n} p_{j} \otimes \omega_{j}$, where $p_{j} \in S^{m-j}(E)$ and $\omega_{j} \in \Gamma\left(\Lambda^{j} M\right)$, then

$$
p_{t}(x, \xi)=\sum_{j=0}^{n} t^{j} p_{j}(x, t \xi) \otimes \omega_{j}
$$

For example, if $p$ is the symbol of a differential operator, then the part of $p$ that is of degree $n$ is multiplied by $t^{n}$.

The technical result which is used to deal with pseudodifferential operators is as follows (Hörmander [13], Widom [18]).

Theorem 3.2. Let $U$ be open in $M$; then for $g \in C^{\infty}(M)$ from a bounded set, with $|d g| \geqq$ $\varepsilon>0$ on $U$, and $u \in \Gamma_{0}(E \mid U)$ from a bounded set, and $p \in S^{*}(E)$, 


$$
\left.e^{-i g(x) / t}\left(\theta p_{t}\right)\left(e^{i g(y) / t} u(y)\right) \sim \sum_{|\alpha| \geqq 0} \frac{t^{|\alpha|}}{\alpha !} \partial_{\xi}^{\alpha} p\left(x, d_{x} g\right) \partial_{v}^{\alpha}\left(e^{i h_{x}(v) / t} \exp _{x}^{*}\left(\tau_{E}(x, y) u(y)\right)\right)\right|_{v=0}
$$

In this formula, $h_{x}(v)=g\left(\exp _{x} v\right)-g(x)-\left\langle v, d_{x} g\right\rangle$; the symbol $\sim$ signifies that the formula is asymptotic in $t$, so that if we take the terms on the right with $|\alpha|<k$, then the error will be $O\left(t^{k / 2}\right)$ in $S^{m-k}(E)$ as $t \rightarrow 0$.

In fact, this is Theorem 3.3 of Hörmander [13], modified by the parameter $t>0$. Hörmander's proofs easily adapt to prove the above theorem.

It follows from Theorem 3.2 that $\sigma$ indeed maps $O p \mathscr{S}^{m}(E)$ to $\mathscr{S}^{m}(E)$, and that for all $N \geqq 0$,

$$
\sigma\left(\theta p_{t}\right)_{t^{-1}}=p+O\left(t^{N}\right) \text { in } \mathscr{S}^{-N}(E) .
$$

Using the family of dilations of symbols, it is possible to define a family of compositions on $\mathscr{S}^{*}(E)$ : if $t>0$, then

$$
\left(p \circ{ }_{t} q\right)_{t}=\theta p_{t} \circ \theta q_{t} .
$$

We can obtain an asymptotic expansion for $p{ }_{t} q$, generalizing the results of Widom [1]. Recall the differential operators $a_{n}$ of Theorem 2.7.

Theorem 3.5. If $p \in \mathscr{S}^{k}(E)$ and $q \in \mathscr{S}^{l}(E)$, then $a_{n}(p, q) \in \mathscr{S}^{k+l-n}(E)$, and we have the asymptotic expansion as $t \rightarrow 0$.

$$
p \circ{ }_{t} q \sim \sum_{n=0}^{\infty} t^{n} a_{n}(p, q)
$$

Proof. Let $h_{y, \xi}(v)=\left\langle\exp _{x}^{-1} \circ \exp _{y} v-\exp _{x}^{-1} y-\left.d \exp _{x}^{-1}\right|_{y} v, \xi\right\rangle$, where $x, y \in M$, $\xi \in T_{x}^{*} M$ and $v \in T_{y} M$ (this is the second order remainder of $\left\langle\exp _{x}^{-1} y, \xi\right\rangle$ around $y$ ). Suppose that $p \in S^{k-r}(E) \otimes \Gamma\left(\Lambda^{r} M\right)$ and $q \in S^{l-s}(E) \otimes \Gamma\left(\Lambda^{s} M\right)$. We will apply Theorem 3.2 to obtain an asymptotic expansion of $\left(p \circ{ }_{t} q\right)_{t}(x, \xi / t)$ :

$$
\begin{aligned}
& \theta p_{t} \circ \theta q_{t}\left(e^{i\left\langle\exp _{x}^{-1} y, \xi\right\rangle / t} \alpha_{\Delta \otimes E}(y, x) u\right) \\
& \sim t^{s} \theta p_{t}\left(\left.e^{i\left\langle\exp _{x}^{-1} y, \xi\right\rangle / t} \sum_{|\alpha| \geqq 0} \frac{t^{|\alpha|}}{\alpha !} \partial_{\eta}^{\alpha} q\left(y,\left(\left.\exp _{x}^{-1}\right|_{y}\right)^{* \xi}+\eta\right)\right|_{\eta=0}\right. \\
& \left.\left.\partial_{v}^{\alpha}\left(e^{i h_{y, \xi}(v) / t} \alpha_{\Delta \otimes E}\left(y, \exp _{y} v\right) \alpha_{\Delta \otimes E}\left(\exp _{y} v, x\right)\right)\right|_{v=0}\right)\left.\right|_{x} u .
\end{aligned}
$$

Applying Theorem 3.2 once more, we obtain

$$
\begin{gathered}
\left(p \circ{ }_{t} q\right)_{t}(x, \xi / t) \sim t^{r+s} \sum_{|\alpha|,|\beta| \geqq 0} \frac{t^{|\alpha|+|\beta|}}{\alpha ! \beta !} \partial_{\xi}^{\beta} p(x, \xi) \circ \partial_{w}^{\beta}\left(\alpha_{\Delta \otimes E}\left(x, \exp _{x} w\right)\right. \\
\left.\partial_{\eta}^{\alpha} q\left(\exp _{x} w,\left(\exp _{x}^{-1} \mid \exp _{x} w\right)^{*} \xi+\eta\right)\right|_{\eta=0} \partial_{v}^{\alpha}\left(e^{i h_{\exp _{x} w \xi}(v) / t}\right. \\
\left.\left.\alpha_{\Delta \otimes E}\left(\exp _{x} w, \exp _{\exp _{x} w} v\right) \alpha_{E}\left(\exp _{\exp _{x} w} v, x\right)\right)\left.\right|_{v=0}\right)\left.\right|_{w=0} u .
\end{gathered}
$$

Since $h_{x, \xi}(0)=0$, it follows that $\partial_{w}^{\beta} \partial_{v}^{\alpha} e^{i h_{\text {exp } p_{x}, \xi}(v) / t}=0\left(t^{-(|\alpha|+|\beta|) / 2}\right)$, so that this formula has the correct homogeneity properties in powers of $t$. (In the above formula, $p(x, \xi) \circ$ means composition of symbols as sections of $\pi^{*} \operatorname{End}(\Delta \otimes E)$.)

The formula we have obtained above shows that $p \circ{ }_{t} q$ is given by an asymptotic sum of differential operators applied to $p \oplus q$; thus, these differential operators may 
be calculated by letting $p$ and $q$ range over the polynomial symbols. But for these symbols, we found the answer in Theorem 2.7. This shows that $a_{n}(p, q) \in \mathscr{S}^{k+l-n}(E)$, which is otherwise not entirely easy to see and identifies $\lim _{t \rightarrow 0} p \circ_{t} q$ as $a_{0}(p, q)$.

Using this theorem, we shall argue below that the symbol of $e^{t^{2} \mathscr{D}^{2}}$, the heat kernel of the square of the Dirac operator, satisfies

$$
\lim _{t \rightarrow 0} \sigma\left(e^{t^{2} \mathscr{D}^{2} / 2}\right)_{t^{-1}}=e^{-\frac{1}{2}|\xi|^{2}+\frac{1}{4} R\left(\xi, \frac{\partial}{\partial \xi}\right)+\frac{1}{32} R \wedge R\left(\frac{\partial}{\partial \xi}, \frac{\partial}{\partial \xi}\right)+\frac{1}{4} F} .
$$

This is well-defined as an element of $\mathscr{S}^{-\infty}(E)$, since $R$ and $F$ are nilpotent. In fact, since $R(\xi, \partial / \partial \xi)$ commutes with the other terms in the exponent, it may be shifted to the right, and is seen not to come into the formula (since $e^{\frac{1}{4} R(\xi, \partial / \partial \xi)} 1=1$.) The proof of the index theorem for the Dirac operator is formally completed by the following formula for $\operatorname{Tr}_{s}(\theta p)$.

Theorem 3.7. If $P \in \operatorname{Op} \mathscr{S}^{-\infty}(E)$, then let $\operatorname{Tr}_{s} P=\left.\operatorname{Tr} P\right|_{\Gamma\left(\Delta^{+} \otimes E\right)}-\left.\operatorname{Tr} P\right|_{\Gamma\left(\Delta^{-} \otimes E\right)}$. If $m<0$, then $\operatorname{Tr}_{s}$ extends by continuity to a linear form on $\mathrm{Op} \mathscr{S}^{m}(E)$, and for all $t>0$,

$$
\operatorname{Tr}_{s} P=(2 \pi)^{-n} \int_{T^{*} M} \operatorname{Tr}_{s}(\sigma P)_{t}(x, \xi) d x d \xi .
$$

(Here, $\operatorname{Tr}_{s} \sigma P$ is computed at each point of $T^{*} M$, and the integration is with respect to the symplectic measure on $\left.T^{*} M.\right)$

Proof. Once the formula is proved for $P \in \mathrm{Op} \mathscr{S}^{-\infty}(E)$, it will be seen to hold for $P \in \mathrm{Op} \mathscr{S}^{m}(E), m<0$, as well, since for such operators, $\sigma P \in \sum_{j=0}^{n} S^{m-j}(E) \otimes \Gamma\left(\Lambda^{j} M\right)$, and only the term in $S^{m-n}(E) \otimes \Gamma\left(\Lambda^{*} M\right)$ gives a contribution to $\operatorname{Tr}_{s} \sigma P$ (Theorem 1.8). Since $S^{m-n}(M)$ is continuously embedded in $L^{1}\left(T^{*} M\right)$, the result follows. satisfy

The proof of the formula is standard, and uses mollification. Let $\rho \in C_{0}^{\infty}\left(\mathbb{R}^{n}\right)$

i) $\rho(x)=1,|x| \leqq \frac{1}{2}$ and $\rho(x) \geqq 0$;

ii) $\rho(x)$ only depends on $|x|$;

iii) $\int \rho(x)=1$.

If $x \in M$ and $\varepsilon>0$ is small enough, then let $\delta_{x, \varepsilon}(y)=\varepsilon^{-n} \rho\left(\varepsilon^{-1} \exp _{x}^{-1} y\right)$. For $u \in \Delta_{x} \otimes E_{x},\left.P\left(\delta_{x, \varepsilon}(y) \alpha_{\Delta \otimes E}(y, x) u\right)\right|_{x} \in \Delta_{x} \otimes E_{x}, \quad$ so that $\left.P \delta_{x, \varepsilon} \alpha_{\Delta \otimes E}(y, x)\right|_{x} \in$ End $\left(\Delta_{x} \otimes E_{x}\right)$. It is clear that

$$
\operatorname{Tr}_{s} P=\left.\lim _{\varepsilon \rightarrow 0} \int_{M} \operatorname{Tr}_{s} P \delta_{x, \varepsilon} \alpha_{\Delta \otimes E}(y, x)\right|_{x}
$$

where the integration is with respect to the Riemannian volume form on $M$. If $\varepsilon$ is small enough that $\delta_{x, \varepsilon}(y) \alpha(y, x)=\delta_{x, \varepsilon}(y)$ for all $x$, then we can write this in terms of $\hat{\rho}$, the Fourier transform of $\rho$, as

$$
\begin{aligned}
\operatorname{Tr}_{s} P & =\left.\lim _{\varepsilon \rightarrow 0}(2 \pi)^{-n} \int_{T^{* M}} \operatorname{Tr}_{s} P\left(e^{i\left\langle\exp _{x}^{-1} y, \xi\right\rangle} \hat{\rho}(\varepsilon \xi) \alpha_{\Delta \otimes E}(y, x)\right)\right|_{x} d \xi d x \\
& =\lim _{\varepsilon \rightarrow 0}(2 \pi)^{-n} \int_{T^{*} M} \hat{\rho}(\varepsilon \xi) \operatorname{Tr}_{s}(\sigma P)(x, \xi) d \xi d x
\end{aligned}
$$


This proves the formula for $t=1$. However, as $t$ varies, the integral is unchanged: although the symbol is dilated by a factor of $t$, giving a factor of $t^{-n}$ after the integral is performed, this is cancelled by the factor of $t^{n}$ that symbols in $S^{m-n},(E) \otimes \Gamma\left(\Lambda^{n} M\right)$ are multiplied by in the definition of $p_{t}$, for $p \in \mathscr{S}^{m}(E)$.

Corollary 3.8. If $p \in \mathscr{S}^{m}(E), m<0$, then

$$
\operatorname{Tr}_{s} \theta p_{t}=(2 \pi)^{-n} \int_{T^{*} M} \operatorname{Tr}_{s} p(x, \xi) d x d \xi+O\left(t^{\infty}\right) .
$$

Here, $O\left(t^{\infty}\right)$ denote $O\left(t^{N}\right)$ for all $N \geqq 0$. The proof follows immediately from (3.3). In the introduction, this formula was interpreted in terms of integration over a symplectic supermanifold; it goes back to Berezin [5] if $M$ is Euclidean.

Combining Theorem 3.7 with (3.6) and (2.11), we have (with $\operatorname{dim} M=2 n$ ),

$$
\text { Ind } \begin{aligned}
\mathscr{D} \mid \Gamma\left(\Delta^{+} \otimes E\right) & =\lim _{t \rightarrow 0} \operatorname{Tr}_{s} e^{t^{2} \mathscr{D}^{2} / 2} \\
= & (2 \pi)^{-2 n} \int_{M} \operatorname{Tr}_{s} e^{F / 4}\left(\operatorname{det} \frac{2 \pi R / 4}{\sin R / 4}\right)^{1 / 2} d x .
\end{aligned}
$$

Using Theorem 1.8 and the definition of $\omega=i^{n} e_{1} \ldots e_{2 n}$, it follows that

$$
\text { Ind } \begin{aligned}
\mathscr{D} \mid \Gamma\left(\Delta^{+} \otimes E\right) & =(2 \pi i)^{-n} \int_{M} \operatorname{Tr}_{E} E^{F / 4}\left(\operatorname{det} \frac{R / 2}{\sin R / 4}\right)^{1 / 2}, \\
& =(2 \pi i)^{-n} 2^{2 n} \int_{M} \operatorname{ch}(-2 \pi i F / 4) \widehat{A}(-2 \pi i R / 4) .
\end{aligned}
$$

Here, we have substituted the definitions of the Chern character, ch $A=\operatorname{tr} e^{-A / 2 \pi i}$, and the $\hat{\mathrm{A}}$-genus, $\hat{\mathrm{A}}(A)=\operatorname{det}((A / 2 \pi i) / \sin (A / 2 \pi i))$. Since the integration over $M$ only gives a non-zero contribution for polynomials in $F$ and $R$ of degree $n$, that is, volume forms, we may cancel the factors of $(2 \pi i)^{-n} 2^{2 n}$, to obtain

$$
\text { Ind } \mathscr{D} \mid \Gamma\left(\Delta^{+} \otimes E\right)=(-1)^{n} \int_{M} \operatorname{ch}(F) \hat{\mathrm{A}}(\mathrm{R}) \text {. }
$$

This is the index theorem for the Dirac operator; the sign is different from that of Atiyah and Singer [4] because of different conventions for Clifford algebras. Specifically, they have a minus sign in the relations that define the Clifford algebra: $x \cdot x=-|x|^{2}$.

It only remains to justify the replacement of $\sigma\left(e^{t^{2} \mathscr{D}^{2}}\right)_{t-1}$ by its top order approximation in (3.6). By standard elliptic theory, $\mathscr{D}^{2}$ has a resolvent for $|\lambda|>\frac{1}{2}$, $|\operatorname{Im} \lambda|>\operatorname{Re} \lambda$ :

$$
\begin{aligned}
& R_{\lambda} \circ\left(\lambda+\mathscr{D}^{2}\right)=\left(\lambda+\mathscr{D}^{2}\right) \circ R_{\lambda}=1, \\
& R_{\lambda}=O(1) \text { in } \operatorname{Op} \mathscr{S}^{-2}(E) .
\end{aligned}
$$

For $t \in(0,1]$, we let $a(t)=t^{2} \sigma\left(\mathscr{D}^{2}\right)_{t^{-1}}$, and $r_{\lambda}(t)=t^{-2}\left(\sigma R_{\lambda t^{-2}}\right)_{t^{-1}}$. The following facts are easy to show:

i) $a(t)=O(1)$ in $\mathscr{S}^{2}(E)$, $\lim _{t \rightarrow 0} a(t)=a(0)=-|\xi|^{2}+\frac{1}{2} F$ 
ii) $r_{\lambda}(t)=O(1)$ in $\mathscr{S}^{-2}(E)$,

$$
\lim _{t \rightarrow 0} r_{\lambda}(t)=r_{\lambda}(0)=\left(\lambda-|\xi|^{2}+\frac{1}{2} R\left(\xi, \frac{\partial}{\partial \xi}\right)+\frac{1}{16} R \wedge R\left(\frac{\partial}{\partial \xi}, \frac{\partial}{\partial \xi}\right)+\frac{1}{2} F\right)^{1},
$$

iii) $\left.\begin{array}{rl}r_{\lambda}(t) \circ{ }_{t}(\lambda+a(t)) \\ (\lambda+a(t)) \circ{ }_{t} r_{\lambda}(t)\end{array}\right\}=1+O\left(t^{N}\right)$ in $\mathscr{S}^{-N}(E) ;$

iv) $\sigma\left(e^{t^{2} \mathscr{D}^{2} / 2}\right)_{t^{-1}}=(-2)^{n} n ! \int_{\Gamma} e^{-\lambda / 2} r_{\lambda}(t)^{n+1} d \lambda$,

where $\Gamma$ is a suitable contour in $\mathbb{C}$, for instance, $|\operatorname{Im} \lambda|=\operatorname{Re} \lambda+1$, oriented counterclockwise.

Clearly, all that remains to be done is to bound $r_{\lambda}(t)^{n+1}-r_{\lambda}(0)^{n+1}$. Indeed,

$$
\begin{aligned}
r_{\lambda}(t)-r_{\lambda}(0)= & r_{\lambda}(0) \circ_{t}\left[(\lambda+a(t)){ }_{t} r_{\lambda}(t)+(a(0)-a(t)){ }_{t} r_{\lambda}(t)-1\right] \\
& +O\left(t^{N}\right) \text { in } \mathscr{S}^{-N}(E) \\
= & r_{\lambda}(0){ }_{t}\left[O(t) \text { in } \mathscr{S}^{-1}(E)\right]=O(t) \text { in } \mathscr{S}^{-3}(E) .
\end{aligned}
$$

Thus,

$$
\begin{aligned}
r_{\lambda}(t)^{n+1}-r_{\lambda}(0)^{n+1} & =\left(r_{\lambda}(t)-r_{\lambda}(0)\right) \sum_{j=0}^{n} r_{\lambda}(t)^{n-j} r_{\lambda}(0)^{j} \\
& =O(t) \text { in } \mathscr{S}^{-2 n-1}(E) .
\end{aligned}
$$

It follows from this that

$$
\begin{aligned}
\sigma\left(e^{t^{2} \mathscr{W}^{2} / 2}\right)_{t-1} & =e^{-\frac{1}{2}|\xi|^{2}+\frac{1}{4} R\left(\xi, \frac{\partial}{\partial \xi}\right)+\frac{1}{32} R \wedge R\left(\frac{\partial}{\partial \xi}, \frac{\partial}{\partial \xi}\right)+\frac{1}{2} F} \\
& +O(t) \text { in } \mathscr{S}^{-\infty}(E) .
\end{aligned}
$$

This estimate proves (3.6), and completes the proof of the index theorem.

\section{References}

1. Alvarez-Gaumé, L.: Supersymmetry and the Atiyah-Singer index theorem. Commun. Math. Phys. 90, 161-173 (1983)

2. Atiyah, M. F., Bott, R.: A Lefschetz fixed point formula for elliptic complexes. II. Ann. Math. 88, 451491 (1968)

3. Atiyah, M. F., Bott, R., Patodi, V. K.: On the heat equation and the index theorem. Inv. Math. 19, 279-330 (1973)

4. Atiyah, M. F., Singer, I. M.: The index of elliptic operators, III. Ann. Math. 87, 546-604 (1968)

5. Berezin, F. A.: The method of second quantization, New York: Academic Press 1966

6. Berezin, F. A., Marinov, M.S.: Particle spin dynamics as the Grassman variant of classical mechanics. Ann. Phys. 104, 336-362 (1977)

7. Bokobza-Haggiag, J.: Opérateurs pseudo-différentiel sur une variété différentiable. Ann. Inst. Four. (Grenoble) 19, 125-177, (1969)

8. Boutet de Mouvel, L.: Hypoelliptic operators with double characteristics and related pseudodifferential operators. Commun. Pure Appl. Math. 27, 585-639 (1976)

9. Bourbaki, N.: Eléments de Mathematique, Algebre, ch. 9., Formes sesquilinéaires et formes quadratiques. Paris: Herman 1959

10. Brauer, R., Weyl, H.: Spinors in $n$ dimensions. Am. J. Math. 57, 425 (1935) 
11. Chevalley, C.: The algebraic theory of spinors. New York: Columbia University, 1954

12. Glimm, J., Jaffe, A.: Quantum physics. New York: Springer 1981

13. Hörmander, L.: Pseudodifferential operators. Commun. Pure Appl. Math. 17, 501-517 (1965)

14. Kostant, B.: Symplectic spinors. Symp. Math, 16, 139-152 (1973)

15. Leites, D. A.: Introduction to the theory of supermanifolds. Usp. Mat. Nauk. 35, 3-57 (1980) (Russ. Math. Surv. 35, 1-64 (1980))

16. Patodi, V. K.: An analytic proof of the Riemann-Roch-Hirzebruch theorem for Kaehler manifolds. J. Diff. Geom. 5, 251-283 (1971)

17. Widom, H.: Families of pseudodifferential operators. In: Topics in Functional analysis. Gohberg, I., and Kac, M. (eds.) New York: Academic Press 1978

18. Widom, H.: A complete symbolic calculus for pseudodifferential operators. Bull. Soc. Math. 104, 19$63(1980)$

19. Witten, E.: Supersymmetry and Morse theory. J. Diff. Geom. 17, 661 (1982)

Communicated by A. Jaffe

Received May 9, 1983 